\title{
Survival outcomes of double- and triple-sequential targeted therapy in patients with metastatic renal cell carcinoma: a retrospective comparison
}

\author{
Sung Han Kim¹, Yoon Seok Suh ${ }^{1}$, Jung Kwon Kim¹, Jae Young Joung ${ }^{1}$, Ho Kyung \\ $\mathrm{Seo}^{1}$, Kang Hyun Lee ${ }^{1}$ and Jinsoo Chung ${ }^{1}$ \\ ${ }^{1}$ Center for Prostate Cancer, Research Institute and Hospital of National Cancer Center, Goyang, Korea \\ Correspondence to: Jinsoo Chung, email: cjs5225@ncc.re.kr \\ Keywords: renal cell carcinoma; metastasis; sequential; targeted therapy; survival \\ Received: May 19, $2017 \quad$ Accepted: August 26, $2017 \quad$ Published: October 19, 2017 \\ Copyright: Kim et al. This is an open-access article distributed under the terms of the Creative Commons Attribution License 3.0 \\ (CC BY 3.0), which permits unrestricted use, distribution, and reproduction in any medium, provided the original author and source \\ are credited.
}

\section{ABSTRACT}

Objective: To evaluate the progression-free survival (PFS) and overall survival (OS) in patients with metastatic renal cell carcinoma (mRCC) treated with doubleand triple-sequence targeted therapy (TT) using tyrosine kinase inhibitors (TKIs) and mammalian target of rapamycin inhibitors (mTORi).

Materials and Methods: Records of 292 patients with mRCC, treated with TT between January 2005 and July 2015, were analyzed retrospectively. Kaplan-Meier and log-rank analyses were used to calculate and compare the total PFS (tPFS) and OS when patients underwent double- or triple-TT using TKIs or mTORi.

Results: Eighty-one (27.7\%) patients who underwent second-line TT were enrolled; $30(\mathbf{1 0 . 3 \% )}$ of whom underwent third-line TT. The tPFS and OS of double-TT using TKI-mTORi ( 5.4 and 30 months, respectively) were significantly better compared with TKI-TKI ( 0.3 and 2 months) or mTORi-TKI ( 2 and 6 months) ( $p<0.001)$. For triple-TT, the tPFS and OS of TKI-mTORi-TKI ( 22.8 and 25 months, respectively) were significantly superior compared with those for TKI-TKI-mTORi (4 and 9 months) ( $p$ $<0.05$ ).

For patients with intermediate-risk according to the Heng or Memorial SloanKettering Cancer Center risk models, TKI-mTORi was associated with a significantly longer tPFS and OS compared with TKI-TKI [expect for OS in the Heng group ( $p=$ 0.086)]. For the triple TT group, TKI-mTORi-TKI resulted in improved tPFS and OS compared with TKI-TKI-TKI or TKI-TKI-mTORi ( $p<0.05)$.

Conclusion: In patients with mRCC, sequential administration of TKI-mTORi led to a significantly superior tPFS compared with any other TT sequence. By contrast, OS did not differ significantly according to TT sequence.

\section{INTRODUCTION}

The advent of multiple targeted therapies (TT) for the treatment of metastatic renal cell carcinoma (mRCC) has renewed hope for increasing the therapeutic response rate, slowing disease progression and improving survival outcomes. Complete responses to treatment are rare, and patients eventually progress, requiring subsequent lines of therapy for disease control [1-3]. Following first-line tumor progression, individualized sequential therapy has become the standard treatment [4-6]. As the number of TTs used for second-, third-, and fourth-line therapies increase, so too do the potential sequential combinations in which they can be administered. For patients with mRCC, the optimal sequence to obtain maximum clinical benefit and improve progressionfree survival (PFS) and overall survival (OS) is unknown. 
Tyrosine kinase inhibitors (TKI) of the vascular epithelial growth factor (VEGF)-receptor and mammalian target of rapamycin inhibitors (mTORi) are the major drug classes used for mRCC treatment. For their anticancer activity, these classes utilize distinct pathways with minimal cross-resistance. Therefore, alternating TT sequentially can improve therapeutic efficacy. The most commonly employed TT sequences are TKI-TKI-mTORi and TKImTORi-TKI [2, 5-9], but there is limited evidence for the optimal sequential TT use for $\mathrm{mRCC}$, especially in Asian patients $[5,6,9]$. This study aimed to compare the survival outcomes of patients who underwent sequential treatment using double- or triple-TT, with or without immunotherapy (ITx). Outcomes were reported as total PFS (tPFS) and OS, according to drug treatment sequence and risk, as classified using the initial prognostic criteria of the Memorial Sloan-Kettering Cancer Center (MSKCC) [10] and the International Metastatic Renal Cell Carcinoma Database Consortium (Heng criteria) risk models [11].

\section{RESULTS}

\section{Baseline patient characteristics}

Between 2005 and 2015, the records for 292 patients with mRCC were included. Eighty-one patients were included in the final analysis. Baseline patient characteristics are shown in Table 1. The median age was 55 years, and patients were predominantly male. Second-, third-, and fourth-line TT were administered to 81 $(27.7 \%), 30(10.3 \%)$, and $9(3.1 \%)$ patients, respectively. Nephrectomy and metastasectomy rates were $28.4 \%$ and $34.6 \%$, respectively. The overall median treatment durations for double- and triple-TT were 30.2 (5.3-66.7) months and $37.8(8.0-83.8)$ months, respectively.

\section{Survival durations according to double- or triple-TT}

The tPFS durations were 10.2 and 17.8 months for patients who underwent double-TT or triple-TT, respectively. The OS durations were 30.0 and 40.0 months for those who underwent double-TT or triple-TT, respectively. Fourteen $(17.3 \%)$ patients remained alive at study completion.

\section{Survival durations according to sequential double-TT}

TKI-mTORi use ( $\mathrm{n}=39,48.1 \%$; tPFS, 15.4 months; OS, 30 months) resulted in superior tPFS durations ( $p$ $<0.001)$ compared with other double-TT regimens as follows: TKI-TKI ( $\mathrm{n}=30,37 \%$; tPFS, 10.3 months; OS, 21 months), mTORi-TKI ( $\mathrm{n}=2,2.5 \%$; tPFS, 5.6 months; OS, 16 months), and TKI-ITx ( $\mathrm{n}=10,12.3 \%$; tPFS, 2 months; OS, 6 months). However, the differences in OS durations were not significant $(\mathrm{p}=0.151)$ (Figure 1).

\section{Survival durations according to sequential triple-TT}

tPFS durations $(\mathrm{p}=0.023)$, but not OS durations $(p=0.235)$, were significantly different according to the triple-TT regimens administered, as follows: TT-TT-ITx (n $=9,29 \%$; tPFS, 33.9 months; OS, 38.0 months), TKI-TKITKI ( $\mathrm{n}=1,3.2 \%$; tPFS, 80.9 months; OS, not available), TKI-mTORi-TKI ( $\mathrm{n}=6,19.4 \%$; tPFS, 22.8 months; OS, 25 months), TKI-TKI-mTORi ( $\mathrm{n}=7,22.6 \%$; tPFS, 14.1 months; OS, 19 months), and TT-ITx-TT ( $\mathrm{n}=8,25.8 \%$; PFS, 8.0; OS, 19.0 months) (Figure 2).

An additional subgroup analysis of PFS for sequential triple-TT to evaluate all five potential triple-TT combinations did not show any significant differences ( $p$ $=0.083$, data not shown), except for the comparison of TKI-TKI-mTORi (2.2 months) and TKI-mTORi-TKI (5.1 months; $p=0.022$, Supplementary Figure 1).

\section{Survival in patients according to double- or triple-TT according to risk}

For the double-TT groups, when stratified by risk classifications, only patients with favorable- or intermediate-risk had significantly different tPFS and OS durations ( $p<0.05$, except for the MSKCC intermediaterisk groups whose OS durations were not significantly different $[\mathrm{p}=0.086]$ ) (Table 2). For the triple-TT groups, significant differences were noted for tPFS in the MSKCC and Heng intermediate risk groups $(\mathrm{p}<0.05)$, whereas there were significant differences for OS in the MSKCC favorable $(p=0.038)$ and Heng intermediate $(p=0.002)$ risk groups. In patients with a poor risk, similar to those in the double-TT groups, there were no significant differences in survival durations ( $\mathrm{p}>0.05$; Table 3 ).

\section{Survival in patients on sequential double-TT according to risk}

For the 56 patients categorized as MSKCC intermediate-risk, tPFS durations for TKI-mTORi, TKITKI, TKI-ITx, and mTORi-ITx groups were 5.4, 10.5, 8.1, and 2.0 months $(\mathrm{p}=0.009)$, and the OS durations were $33,26,18$, and 5.0 months $(\mathrm{p}=0.086)$, respectively. For the 50 patients categorized as Heng intermediate-risk, tPFS durations for TKI-mTORi, TKI-TKI, TKI-ITx, and mTORi-ITx were 15.4, 10.8, 8.1, and 2 months, and OS durations were $33,26,18$, and 5 months, respectively (Figure 3).

\section{Survival in patients on sequential triple-TT according to risk}

For MSKCC intermediate-risk patients $(\mathrm{n}=15)$, the tPFS durations for TT-TT-ITx, TKI-mTORi-TKI, ITxTT-TT, and TKI-TKI-TKI groups were 23.3, 15.9, 14.1, 
Table 1: Baseline patient characteristics $(\mathbf{N}=\mathbf{8 1})$

\begin{tabular}{|c|c|}
\hline & N(\%) or Median (min-max) \\
\hline Age (years) & $55(30-76)$ \\
\hline gender (Male/ Female) & $64 / 17(79 / 21)$ \\
\hline Nephrectomy/metastasectomy & $22 / 28(28.4 / 34.6)$ \\
\hline Heng favorable risk & $15(18.5)$ \\
\hline Intermediate risk & $60(74.1)$ \\
\hline Poor risk & $6(7.4)$ \\
\hline MSKCC favorable risk & $18(22.2)$ \\
\hline Intermediate risk & $56(69.1)$ \\
\hline Poor risk & $7(8.6)$ \\
\hline Pathologic/clinical T, T2, T3, T4 & 23/12/35/11 (13.5/28.4/14.8/43.2) \\
\hline N0, N, Nx & $20 / 19 / 42(24.7 / 23.5 / 51.8)$ \\
\hline M & $54(66.7)$ \\
\hline Fuhrman nuclear grade 1/2/3/4/unknown & $8 / 23 / 24 / 3 / 23(9.9 / 28.4 / 29.6 / 3.7 / 28.4)$ \\
\hline Histology Clear cell/ Non-clear cell/unknown & $68 / 7 / 6(84 / 8.6 / 7.4)$ \\
\hline Second line ITx/TKI/mTORi (N=81) & $10 / 35 / 36(12.3 / 43.2 / 44.5)$ \\
\hline Third line ITx/TKI/mTORi (N=30) & $5 / 16 / 9(16.7 / 53.3 / 30)$ \\
\hline Forth line TT & $9(11.1)$ \\
\hline \multicolumn{2}{|l|}{ Double Sequential TT } \\
\hline TKI-mTORi & $39(48.1)$ \\
\hline TKI-TKI & $30(37.1)$ \\
\hline TKI-ITx & $10(12.3)$ \\
\hline mTORi-TKI & $2(2.5)$ \\
\hline \multicolumn{2}{|l|}{ Double sequence response-RECIST } \\
\hline $\mathrm{CR} / \mathrm{PR} / \mathrm{SD} / \mathrm{PD}$ & $0 / 6 / 48 / 27(0 / 7.4 / 59.3 / 33.3)$ \\
\hline \multicolumn{2}{|l|}{ Triple sequential TT } \\
\hline TKI-TKI-TKI & $1(3.3)$ \\
\hline TT-TT-ITx & $9(30.0)$ \\
\hline ITx-TT-TT & $8(26.7)$ \\
\hline TKI-mTORi-TKI & $6(20.0)$ \\
\hline TKI-TKI-mTORi & $6(20.0)$ \\
\hline \multicolumn{2}{|l|}{ Triple sequence response-RECIST } \\
\hline $\mathrm{CR} / \mathrm{PR} / \mathrm{SD} / \mathrm{PD}$ & $0 / 5 / 17 / 8(0 / 16.6 / 56.7 / 26.7)$ \\
\hline Treatment duration of Second/Third-line TT(Month) & $3.1(1-66.7) / 5.0(1-47.1)$ \\
\hline Overall median duration of double/triple sequential TT (months) & $30.2(5.3-66.7) / 37.8(8-83.8)$ \\
\hline Total PFS of Double/Triple sequential TT (Month) & $10.2(1-74.4) / 17.8(3.5-83.8)$ \\
\hline OS of Double/Triple sequential TT (Months) & $30.0(21.1-38.8) / 40.0(18.4-61.6)$ \\
\hline Survival/death & $14 / 67(17.3 / 82.7)$ \\
\hline
\end{tabular}

TT, targeted therapy; ITx, immunotherapy; TKI, tyrosine kinase inhibitor; mTORi, mammalian target of rapamycin inhibitor; CR, complete remission; PR, partial response; SD, stable disease; PD, progressive disease; PFS, progression-free survival; OS, overall survival 
and 8 months, respectively. For the same patients, the OS durations for TT-TT-ITx, TKI-mTORi-TKI, ITx-TT-TT, and TKI-TKI-mTORi were 33, 29, 40, and 12 months (p $>0.05$ ), respectively.

For Heng intermediate-risk patients $(n=16)$, the tPFS durations for TKI-mTORi-TKI, TT-TT-ITx, ITxTT-TT, and TKI-TKI-mTORi were 33.9, 23.3, 16.7, and 8 months, respectively, while the OS durations for

A

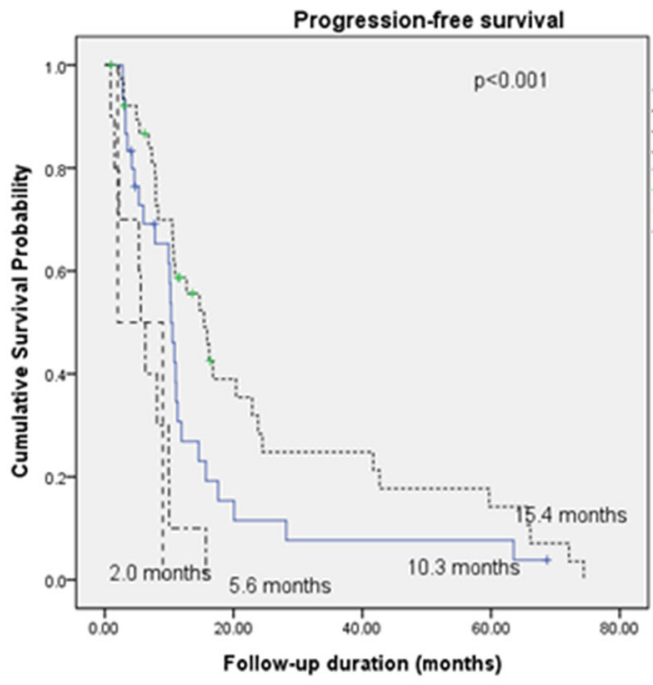

TKI-mTORi-TK, ITx-TT-TT, TT-TT-ITx, and TKI-TKImTORi were 53, 49, 33, and 12 months, respectively (p $<0.05$ ) (Figure 4).

\section{DISCUSSION}

TT has considerably improved prognosis in patients with mRCC with an approximate doubling of median OS
B

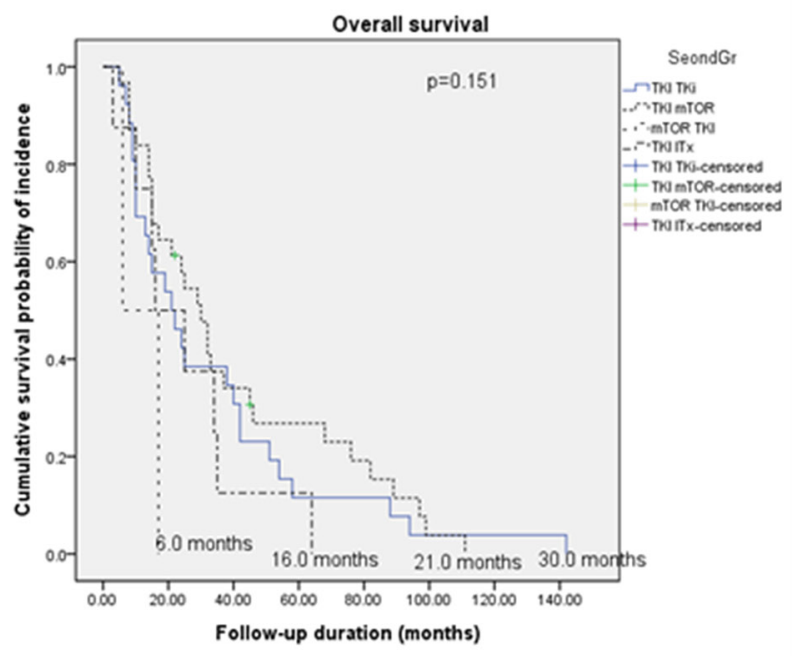

Figure 1: Kaplan-Meier curves of (A) progression-free survival and (B) overall survival according to double sequential targeted therapy.

A

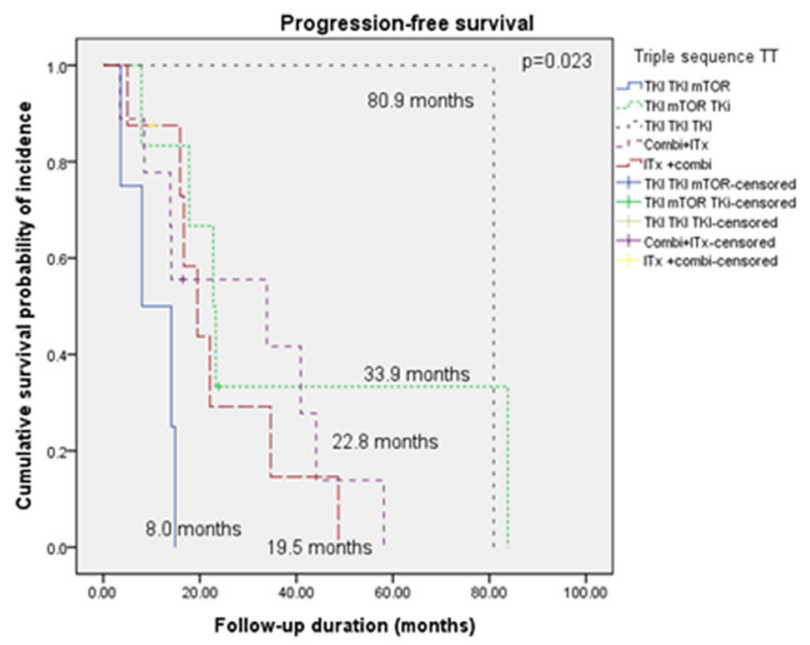

B

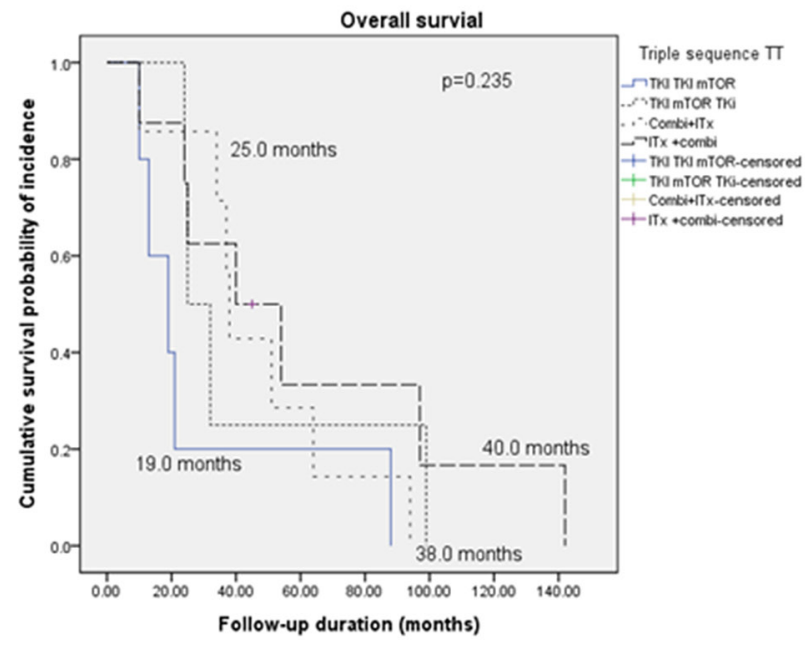

Figure 2: Kaplan-Meier curves of (A) progression-free survival and (B) overall survival according to triple sequential targeted therapy. 
Table 2: Comparison of total progression-free survival and overall survival of double sequence according to the prognostic models of the MSKCC and Heng criteria

\begin{tabular}{lccc}
\hline $\begin{array}{l}\text { MSKCC/Heng } \\
\text { criteria }\end{array}$ & Favorable (mos., 95\% CI) & Intermediate (mos., 95\% C. I.) & Poor (mos., 95\% C.I.) \\
\hline PFS (p-value) & $<0.001 / 0.002$ & $0.009 / 0.003$ & $0.768 / 0.636$ \\
TKI-mTORi & $42.7(17.8-67.6)$ & $15.4(8.8-22.0) / 15.4(6.6-24.2)$ & $2.9(1.7-6.6)$ \\
TKI-TKI & $10.2(1-24.0) / 10.2(3.5-16.9)$ & $10.5(9.6-11.4) / 10.8(9.8-11.8)$ & $5.3(2.4-12.3) / 4.6(3.2-12.7)$ \\
TKI-ITx & $5.6(1-12.1)$ & $8.1(1-17.3) / 8.1(1.0-15.3)$ & $5.3(3.3-8.5) /$ NA \\
& NA & $2.0(1-12.4)$ & NA \\
mTORi -TKI & $0.024 / 0.046$ & $0.086 / 0.048$ & $0.737 / 0.863$ \\
OS (p-value) & $115(104.7-125.3) / 125(1-275.4)$ & $33(19.6-46.4)$ & $8{ }^{*}$ \\
TKI- mTORi & $67(1-159.1) / 115(104.7-125.3)$ & $16(19.6-46.4)$ & $(4.1-11.9) / 9(4.1-13.9)$ \\
TKI-TKI & $14.0(1-62.8)$ & $18.0(7.2-28.8)$ & $16^{*}$ \\
TKI-ITx & NA & $5.0(1-21.3)$ & NA \\
mTORi -TKI & 5 & & \\
\hline
\end{tabular}

*. only one patient's clinical outcome

C.I, confidence interval; PFS, progression-free survival; OS, overall survival

Table 3: Comparison of total progression-free survival and overall survival of triple sequences according to prognostic models of the MSKCC and Heng criteria

\begin{tabular}{lccc}
\hline $\begin{array}{l}\text { MSKCC/ Heng } \\
\text { criteria }\end{array}$ & Favorable (mos., 95\% CI) & Intermediate (mos., 95\%C.I.) & Poor (mos., 95\%C.I.) \\
\hline PFS (p-value) & $0.183 / 0.248$ & $0.041 / 0.012$ & $0.317 / 0.273$ \\
TT-TT-ITx & $33.9(1-74.5) / 8.5(1-56.5)$ & $14.1(1-43.1) / 33.9(1-74.5)$ & NA \\
ITX-TT-TT & $22.1(17.9-26.3) / 22.1(9.3-61.5)$ & $15.9(4.4-27.4) / 16.7(13.2-20.2)$ & $5^{*}$ \\
TKI-mTORi-TKI & $17.8^{*}$ & $23.3(1-63.2)$ & $7.9^{*}$ \\
TKI-TKI-mTORi & NA & $8.0(1.0-15.0)$ & $14.1^{*}$ \\
OS (p-value) & $0.030 / 0.090$ & $0.078 / 0.006$ & NA/ 0.317 \\
TT-TT-ITx & $67(35.0-99.0) / 47.0(9.6-162.4)$ & $40.0(1-79.2) / 49(19.7-63.3)$ & NA \\
ITX-TT-TT & $76(1-154.4) / 111.0(38.6-175.4)$ & $29(1-71.1) / 53(6.9-99.1)$ & NA/ $10^{*}$ \\
TKI-mTORi-TKI & $25^{*}$ & $33(18.6-47.4)$ & NA \\
TKI-TKI-mTORi & $88^{*}$ & $12.0(7.2-16.8)$ & NA/ $21^{*}$ \\
\hline
\end{tabular}

*, only one patient's outcome

C.I, confidence interval; PFS, progression-free survival; OS, overall survival

since the immunotherapy era [12]. Multiple randomized, controlled, clinical trials of single TT have established the objective therapeutic response rate, PFS, and OS. For maximum clinical benefit, TT should be used sequentially and tailored to the patient's needs. However, despite multiple guidelines with established positions on the sequential use of TT, the optimal combination is unclear $[5,6]$.

For mRCC, the underlying concepts of sequential TT are to prevent treatment resistance and augment anti-tumor activity. Tumor cells can develop treatment resistance via upregulation of pro-angiogenic signals (e.g., placental growth factor or fibroblast growth factor) or by increased expression of protective pericytes that express the platelet-derived growth factor receptor. These crucial mechanisms can be more efficiently inhibited using multi-targeted TKIs, compared with administration of everolimus or bevacizumab alone [3, 13]. Following progression during TKI administration, another concept is to switch to an mTORi, which operate via a different pharmacodynamic profile to augment antitumoral effects. 
In the present study, the tPFS and OS of each drug sequence was compared, and patients who were treated with TKI-mTORi, had a significantly longer tPFS, and a numerically higher (although not statistically significant) OS compared with those who were administered TKI-TKI (Figure 1, Supplementary Figure 2). This finding is similar to that of an observational meta-analysis conducted by Heng et al., which compared the PFS and OS of secondline mRCC treatment using an mTORi versus a TKI [4, 14]. They showed that second-line use of an mTORi was associated with significantly prolonged survival, compared to the second-line use of a TKI. Based on this evidence, the optimal treatment sequence dilemma of whether to shift to an mTORi or to persevere with angiogenesis inhibition (VEGF-TKI), is one of the main unresolved investigational issues for patients with $\operatorname{mRCC}[15,16]$.
Current guidelines for $\mathrm{mRCC}$ are of limited value in selecting $\mathrm{TT}$, because of heterogeneity between patients, an almost complete absence of evidence-based data on sequential therapy, and the lack of predictive biomarkers for the TT approved for $\operatorname{RCC}[5,6]$. According to evidence-based guidelines, most patients with mRCC are initially treated with TKI, except patients with an unfavorable prognosis who might be prescribed temsirolimus [17]. The optimal treatment choice following the failure of first-line TKI remains a matter of debate since head-to-head, prospective clinical studies comparing the efficacy between a TKI and an mTORi in patients who have failed initial TKI therapy have not been conducted $[17,18]$. Therefore, dissemination of data regarding practice-oriented $\mathrm{TT}$ use in patients with $\mathrm{mRCC}$ is desirable and of increasing importance.
A

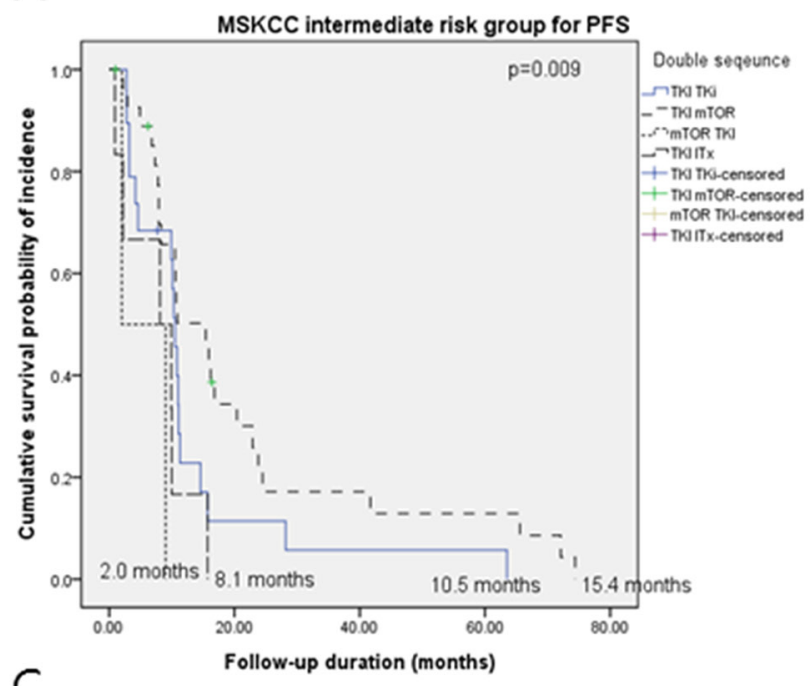

C

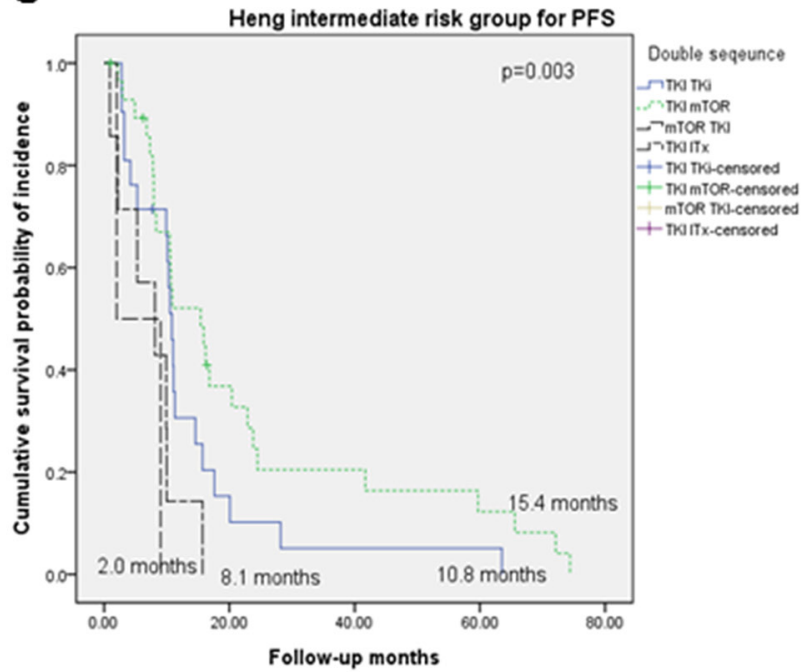

B
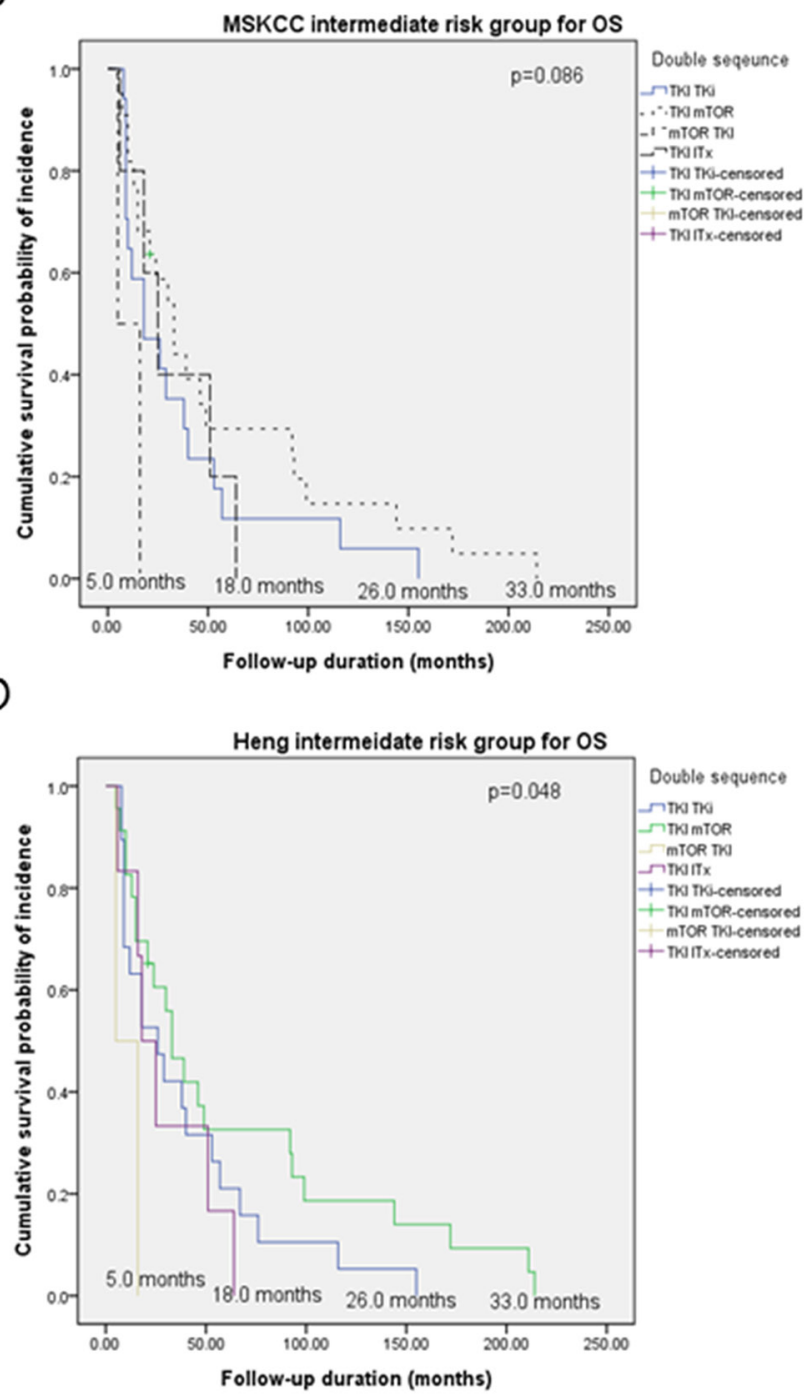

Figure 3: Kaplan-Meier curves of $(\mathbf{A}, \mathbf{C})$ progression-free survival and $(\mathbf{B}, \mathbf{D})$ overall survival according to triple sequential targeted therapy in $\mathrm{mRCC}$ patients with intermediate MSKCC and Heng risks. 
The choice between either a TKI or an mTORi for the subsequent, second-line TT should be based on toxicity profile, efficacy, and tolerability, as well as potential cross-resistance between the first and second-line TT. The AXIS clinical trial, which compared the effectiveness of axitinib versus sorafenib in advanced RCC, did not show absolute cross-resistance between TKIs [19]; however, the sequential use of two pharmacodynamically similar TKIs could be associated with cumulative gastrointestinal side effects.

Escudier et al. [17] suggested that rapid therapy switching should be avoided for patients with slow disease progression, with a switch performed when progression occurred. Such an approach would keep other therapeutic options available for as long as possible [20]. For rapidly progressing disease, however, a rapid switch should be considered as early as possible. In the cases where tumor response is mixed, e.g. stabilization in one lesion but progression in another, and when there is evidence of new metastases, isolated progressive tumors or metastases should be treated with local surgery and radiotherapy [6]. However, in the case of relevant progression, a switch in systemic therapy is necessary. Furthermore, a switch is obligatory if there is unacceptably high toxicity, or if the conservative management of adverse events fails.

For third-line sequential TT, in the present study, TKI-mTORi-TKI doubled OS compared with TKI-TKImTORi. This finding is dissimilar to that of Iacoville et al. who indicated that TKI-TKI-mTORi resulted in superior outcomes compared with TKI-mTORi-TKI [8]. Such discrepancies could be due to the heterogeneity of data sources, confounding factors, selection bias due to the lack of randomization, and the small number of cases in the present study. In the triple-TT group, there were only
A

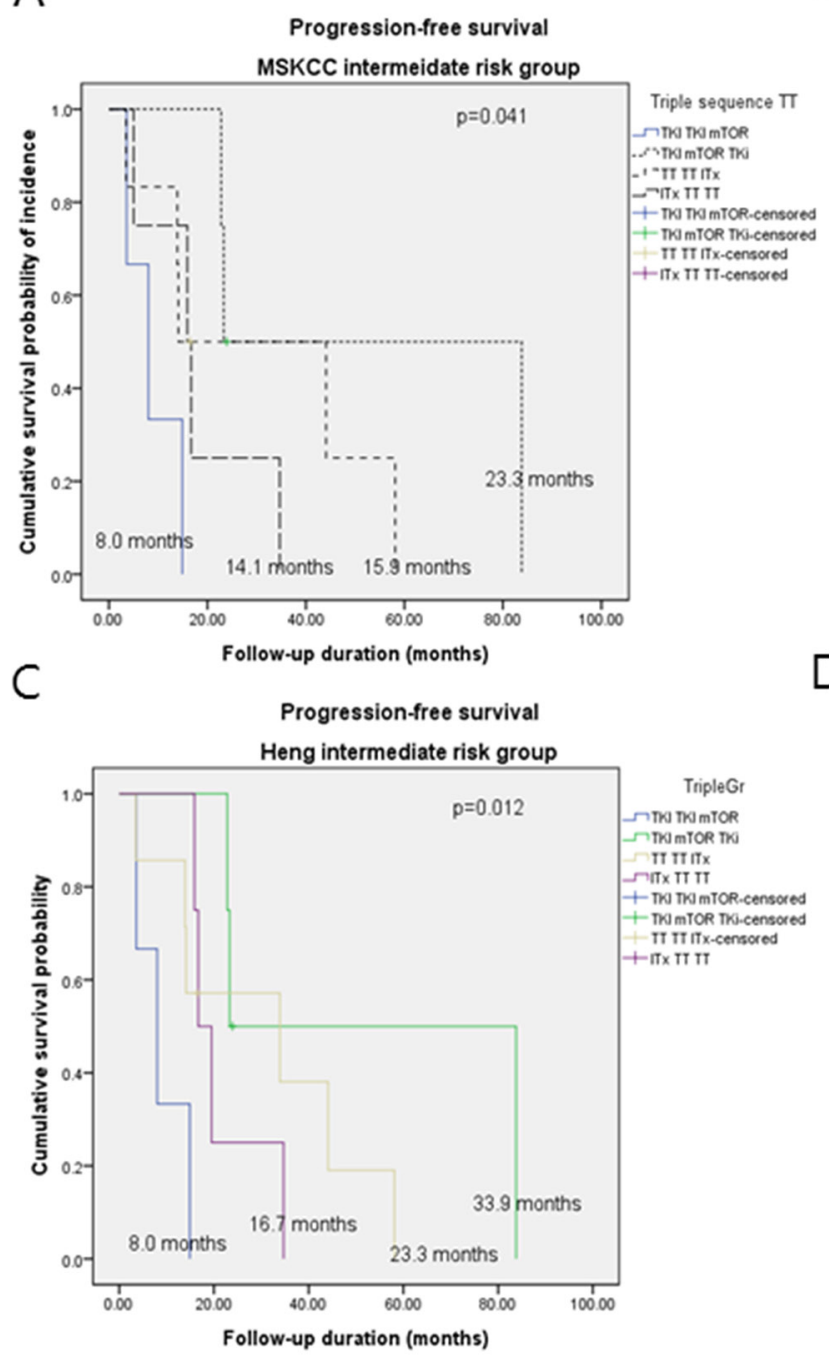

B
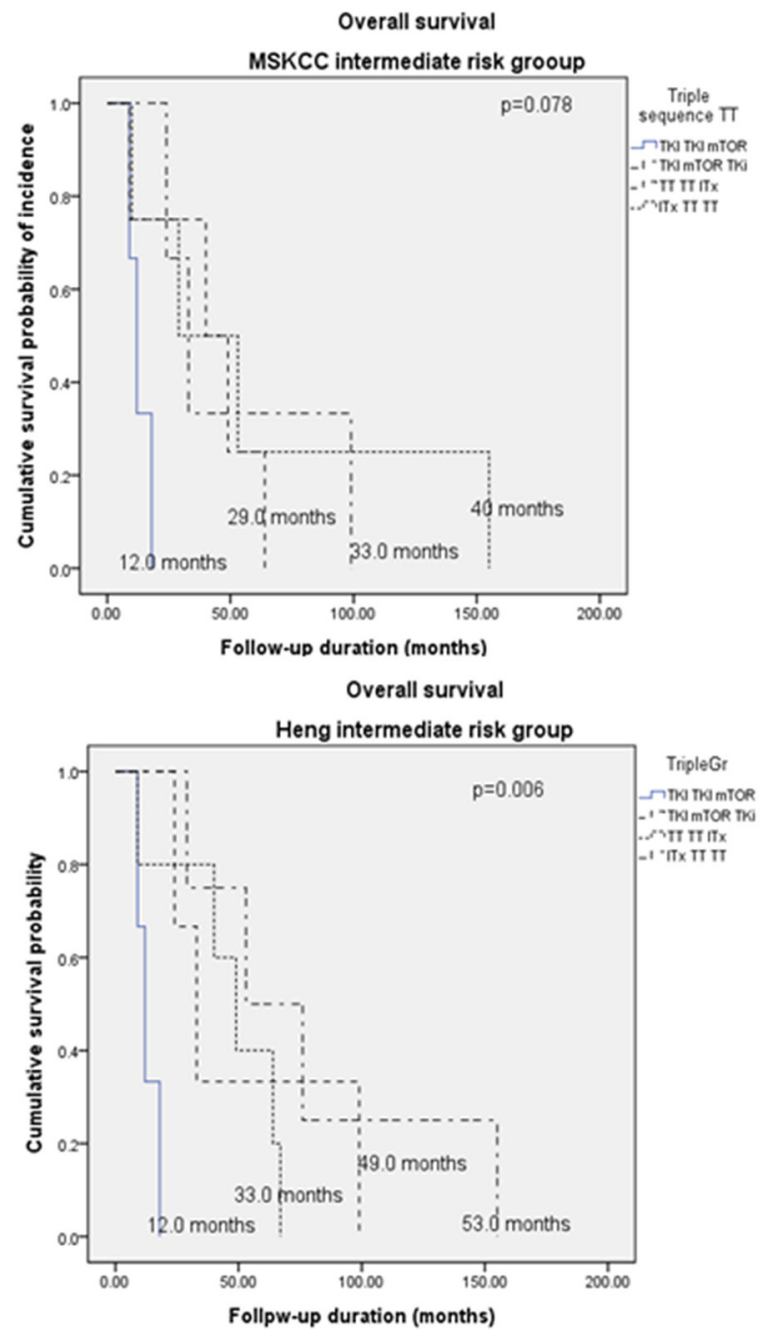

Figure 4: Kaplan-Meier curves of $(\mathbf{A}, \mathbf{C})$ progression-free survival and $(\mathbf{B}, \mathbf{D})$ overall survival according to triple sequential targeted therapy in mRCC patients with intermediate MSKCC and Heng risks. 
2 patients with favorable- and poor-risk classifications, and the remainder were classified as intermediate-risk. The proportion of poor-risk patients in Iacoville et al.'s study $(6 \%)$ was similar to that in the present study $(8.6 \%)$. However, the proportion of favorable-risk patients was much higher in that study (46\%) compared with the present study $(22.2 \%)$ suggesting that the sequential combinations including a second-line mTORi might be associated with more favorable outcomes. By contrast, and in agreement with the present study, Heng et al. found that TKI-mTORi-TKI had superior outcomes, similar to the finding that TKI-mTORi had significantly better outcomes compared to use of two successive TKIs [14].

A subanalysis comparing all combinations of thirdline TTs showed that the PFS associated with triple TT was significantly lower in patients treated with TKI-TKImTORi compared to that in those treated TKI-mTORiTKI. These findings suggest that third-line TT using a TKI might be better tolerated and associated with improved quality of life compared with a third-line mTORi [21]. Such superiority of TKI-mTORi-TKI compared with TKI-TKI-mTORi might be because the use of pazopanib as a third-line TKI might inhibit not only VEGF pathways but other alternative angiogenesis pathways [22, 23]. Lastly, the cross-resistance between similar TKIs and the differential modes of action might affect the superiority of TKI-mTORi-TKI [24].

In the present study, ITx was also used as a secondand third-line drug. The TT-TT-ITx and ITx-TT-TT groups had favorable treatment outcomes compared with other triple-TT groups. Recent reports have raised the possibility of a potential immunomodulatory effect of TT $[25,26]$, and data underlining the effect of mTORi on anticancer immunity have been presented [27]. Recent randomized clinical trials with new target agents, in the second- or third-line setting, demonstrated better efficacy than second-line everolimus for TKIfailed mRCC patients [28]. OS values after treatment with cabozantinib (21.4 months), and lenvatinib with everolimus (14.6 months) were significantly better compared with everolimus alone (18.8 and 5.5 months, respectively) [28]. An immune checkpoint inhibitor, called nivolumab has shown improvement of OS compared with everolimus in the second- and thirdline setting [29]. These new agents are likely to further change the approach to sequential and combinational TT treatment in mRCC.

Subgroup analyses based on prognostic risk showed variable prognostic outcomes between the double- and triple-TT. The discordance in survival outcomes, according to the risk models, in patients with an intermediate risk, might be explained by the small numbers of patients, which could have an impact on the risk assessment variables used to determine intermediate risk between the two models [20]. The MSKCC model was developed during the immunotherapeutic era for patients undergoing
ITx and has been shown to have limitations, and a weaker predictive ability for survival in patients treated using TT, compared with the Heng model.

The present study has limitations. It was a retrospective study in selected patients, who were healthy enough to be treated using triple-TT. The relatively low numbers of patients included for each TT combination can be seen as hypothesis-generating, but does not allow any definitive conclusions to be made regarding the optimal TT sequence. Other factors such as patient preference, comorbidities, tumor burden, symptoms, and different prognostic models need to be considered when making treatment decisions. Also, the treatment cost and availability, depending on the health insurance system of each country, should be considered. Future large-scale, prospective studies with a greater number of patients undergoing each treatment type are needed to gain a better insight into the optimal TT sequence in patients with mRCC.

In conclusion, the present study did show that TKI-mTORi sequential therapy resulted in significantly superior tPFS compared with other double-TT therapies, whereas OS was not dependent on the combination of sequential TT. The combination of sequential TT with ITx also showed favorable tPFS and OS.

\section{MATERIALS AND METHODS}

\section{Ethics statement}

This retrospective study was approved by the Institutional Review Board (IRB) of the National Cancer Center (IRB No. NCC206-0262). The need for written consent was waived. Patient data were anonymized and de-identified before analysis. Study procedures were performed in accordance with the ethics of the Declaration of Helsinki.

\section{Eligibility criteria}

The records of patients with $\mathrm{mRCC}$, treated between January 2005 and July 2015 with a TT, were collected from a prospective RCC database. Patients with non-measurable disease, or no follow-up records, were excluded. Initial risk classifications based on the MSKCC [10] and Heng risk criteria [11] were used to predict response to doubleand triple-TT.

Fuhrman nuclear grade and the Tumor-NodeMetastases staging (Union for International Cancer Control, 2009) classification were used to evaluate RCC pathologically [30]. Treatment response was measured according to the Response Evaluation Criteria in Solid Tumors (RECIST, version 1.1) [31] using computed tomography or magnetic resonance imaging performed according to local procedures (between every 8-12 weeks). Disease progression was defined as $\mathrm{a} \geq 20 \%$ increase in the 
longest diameter, as per RECIST, or the development of any new metastatic lesion.

The choice of TT sequence was decided by the treating clinician (JC), guided by the patient's pathology and coverage by National Insurance [32]. Standard dosage reductions, in cases of toxicity, were managed by the treating physician. TT was classified into two groups, based on the mechanism of action: TKIs (axitinib, pazopanib, sorafenib, and sunitinib) or mTORis (everolimus and temsirolimus). In addition, some patients underwent interferon-alpha ITx during second- or thirdline treatment. All agents were administered in accordance with manufacturers' instructions.

\section{Statistical analysis}

The tPFS and OS durations following double- or triple-TT were the primary outcomes. Differences and associations between the baseline characteristics were examined using the Chi-square test, Fisher's exact test, and the Kruskal-Wallis test, as appropriate. The tPFS was defined as the sum of PFS time for each double- and third-line TT, from the date of TT initiation to the date of disease progression, from any cause. OS was defined as the period from treatment initiation to death by any cause or to the last contact with the patient. Statistical analyses of tPFS and OS for each combination of TT were evaluated according to the MSKCC and Heng risk criteria using Kaplan-Meier curves with the log-rank test. All results were considered statistically significant if the twosided $\mathrm{p}$ values were $<0.05$ using SAS 9.2 software (SAS Institute Inc., Cary, NC, USA).

\section{Abbreviations}

mRCC, metastatic renal cell carcinoma

TKI, tyrosine kinase inhibitor

mTORi, mammalian target of rapamycin inhibitor

TT, targeted therapy

ITx, immunotherapy

PFS, progression-free survival

tPFS, total progression-free survival

OS, overall survival

\section{Author contributions}

SH Kim collected the data and wrote the manuscript.

J Chung designed the study, treated the patients, and reviewed the manuscript

YS Sub, JK Jung, JY Joung, HK Seo, J Chung, and $\mathrm{KH}$ Lee participated in the collection of the cases and helped to draft and review the manuscript.

\section{CONFLICTS OF INTEREST}

All the authors have no conflicts of interest.

\section{FINANCIAL DISCLOSURE}

This work was supported by grants from the National Cancer Center (No. NCC1710290-1), The Republic of Korea.

\section{REFERENCES}

1. Harshman LC, Xie W, Bjarnason GA, Knox JJ, MacKenzie M, Wood L, Srinivas S, Vaishampayan UN, Tan MH, Rha SY, Donskov F, Agarwal N, Kollmannsberger C, et al. Conditional survival of patients with metastatic renalcell carcinoma treated with VEGF-targeted therapy: a population-based study. Lancet Oncol. 2012; 13: 927-35.

2. Hutson TE, Bukowski RM, Cowey CL, Figlin R, Escudier $\mathrm{B}$, Sternberg CN. Sequential use of targeted agents in the treatment of renal cell carcinoma. Crit Rev Oncol Hematol. 2011; 77: 48-62.

3. Procopio G, Verzoni E, Iacovelli R, Guadalupi V, Gelsomino F, Buzzoni R. Targeted therapies used sequentially in metastatic renal cell cancer: overall results from a large experience. Expert Rev Anticancer Ther. 2011; 11: $1631-40$.

4. Porta C, Szczylik C, Escudier B. Combination or sequencing strategies to improve the outcome of metastatic renal cell carcinoma patients: a critical review. Crit Rev Oncol Hematol. 2012; 82: 323-37.

5. Albiges L, Choueiri T, Escudier B, Galsky M, George D, Hofmann F, Lam T, Motzer R, Mulders P, Porta C, Powles T, Sternberg C, Bex A. A systematic review of sequencing and combinations of systemic therapy in metastatic renal cancer. Eur Urol. 2015; 67: 100-10.

6. Bergmann L, Beck J, Bothe K, Brinkmann OA, Buse S, Goebell PJ, Grünwald V, Holzapfel K, Kübler H, Marschner NW, Mickisch G, Schultze-Seemann W, Siebels M, et al. Treatment algorithm for metastatic renal cell carcinoma-recommendations based on evidence and clinical practice. Oncol Res Treat. 2014; 37: 136-41.

7. Porta C, Procopio G, Cartenì G, Sabbatini R, Bearz A, Chiappino I, Ruggeri EM, Re GL, Ricotta R, Zustovich F, Landi L, Calcagno A, Imarisio I, et al. Sequential use of sorafenib and sunitinib in advanced renal-cell carcinoma (RCC): an Italian multicentre retrospective analysis of 189 patient cases. BJU Int. 2011; 108: E250-7.

8. Iacovelli R, Cartenì G, Sternberg CN, Milella M, Santoni M, Di Lorenzo G, Ortega C, Sabbatini R, Ricotta R, Messina C, Lorusso V, Atzori F, De Vincenzo F, et al. Clinical outcomes in patients receiving three lines of targeted therapy for metastatic renal cell carcinoma: results from a large patient cohort. Eur J Cancer. 2013; 49: 2134-42.

9. Kumano M, Miyake H, Harada K, Fujisawa M. Sequential use of mammalian target of rapamycin inhibitors in patients with metastatic renal cell carcinoma following failure of tyrosine kinase inhibitors. Med Oncol. 2013; 30: 745. 
10. Motzer RJ, Bacik J, Mazumdar M. Prognostic factors for survival of patients with stage IV renal cell carcinoma: memorial sloan-kettering cancer center experience. Clin Cancer Res. 2004; 10: 6302S-3S.

11. Heng DY, Xie W, Regan MM, Warren MA, Golshayan AR, Sahi C, Eigl BJ, Ruether JD, Cheng T, North S, Venner P, Knox JJ, Chi KN, et al. Prognostic factors for overall survival in patients with metastatic renal cell carcinoma treated with vascular endothelial growth factor-targeted agents: results from a large, multicenter study. J Clin Oncol. 2009; 27: 5794-9.

12. Escudier B, Eisen T, Porta C, Patard JJ, Khoo V, Algaba F, Mulders P, Kataja V; ESMO Guidelines Working Group. Renal cell carcinoma: ESMO Clinical Practice Guidelines for diagnosis, treatment and follow-up. Ann Oncol. 2012; 23: vii65-71.

13. Bergers G, Hanahan D. Modes of resistance to antiangiogenic therapy. Nat Rev Cancer. 2008; 8: 592-603.

14. Heng DY, Signorovitch J, Swallow E, Li N, Zhong Y, Qin P, Zhuo DY, Wang X, Park J, Stergiopoulos S, Kollmannsberger C. Comparative effectiveness of secondline targeted therapies for metastatic renal cell carcinoma: a systematic review and meta-analysis of real-world observational studies. PloS One. 2014; 9: e114264.

15. Porta C. Entropy increases in kidney cancer treatment, but a bit of simplicity may emerge from chaos. Eur Urol. 2011; 60: 1171-2.

16. Iacovelli R, Palazzo A, Trenta P, Mezi S, Pellegrino D, Naso G, Cortesi E. Management of metastatic renal cell carcinoma progressed after sunitinib or another antiangiogenic treatment. Am J Clin Oncol. 2014; 37: 611-5.

17. Escudier B, Szczylik C, Porta C, Gore M. Treatment selection in metastatic renal cell carcinoma: expert consensus. Nat Rev Clin Oncol. 2012; 9: 327-37.

18. Calvo E, Ravaud A, Bellmunt J. What is the optimal therapy for patients with metastatic renal cell carcinoma who progress on an initial VEGFr-TKI? Cancer Treat Rev. 2013; 39: 366-74.

19. Rini BI, Escudier B, Tomczak P, Kaprin A, Szczylik C, Hutson TE, Michaelson MD, Gorbunova VA, Gore ME, Rusakov IG, Negrier S, Ou YC, Castellano D, et al. Comparative effectiveness of axitinib versus sorafenib in advanced renal cell carcinoma (AXIS): a randomised phase 3 trial. Lancet. 2011; 378: 1931-9.

20. Kwon WA, Cho IC, Yu A, Nam BH, Joung JY, Seo HK, Lee KH, Chung J. Validation of the MSKCC and Heng risk criteria models for predicting survival in patients with metastatic renal cell carcinoma treated with sunitinib. Ann Surg Oncol. 2013; 20: 4397-404.

21. Calvo E, Schmidinger M, Heng DY, Grünwald V, Escudier B. Improvement in survival end points of patients with metastatic renal cell carcinoma through sequential targeted therapy. Cancer Treat Rev. 2016; 50: 109-117.

22. Pal SK, Hossain DM, Zhang Q, Frankel PH, Jones JO, Carmichael C, Ruel C, Lau C, Kortylewski M. Pazopanib as third line therapy for metastatic renal cell carcinoma: clinical efficacy and temporal analysis of cytokine profile. J Urol. 2015; 193: 1114-1121.
23. Matrana MR, Baiomy A, Campbell M, Alamri S, Shetty A, Teegavarapu P, Kalra S, Xiao L, Atkinson B, Corn P, Jonasch E, Elsayes KM, Tannir NM. Outcomes of patients with metastatic clear-cell renal cell carcinoma treated with pazopanib after disease progression with other targeted therapies. Eur J Cancer. 2013; 49: 3169-3175.

24. Eichelberg C, Vervenne WL, De Santis M, Fischer von Weikersthal L, Goebell PJ, Lerchenmüller C, Zimmermann U, Bos MM, Freier W, Schirrmacher-Memmel S, Staehler M, Pahernik S, Los M, et al. SWITCH: A randomised, sequential, open-label study to evaluate the efficacy and safety of sorafenib-sunitinib versus sunitinib-sorafenib in the treatment of metastatic renal cell cancer. Eur Urol. 2015; 68: 837-847.

25. Adotevi O, Pere H, Ravel P, Haicheur N, Badoual C, Merillon N, Medioni J, Peyrard S, Roncelin S, Verkarre V, Mejean A, Fridman WH, Oudard S, et al. A decrease of regulatory $\mathrm{T}$ cells correlates with overall survival after sunitinib-based antiangiogenic therapy in metastatic renal cancer patients. J Immunother. 2010; 33: 991-8.

26. Kobayashi M, Kubo T, Komatsu K, Fujisaki A, Terauchi F, Natsui S, Nukui A, Kurokawa S, Morita T. Changes in peripheral blood immune cells: their prognostic significance in metastatic renal cell carcinoma patients treated with molecular targeted therapy. Med Oncol. 2013; 30: 556.

27. Thiery-Vuillemin A, Theodore C, Jacobasch L, Schmitz J, Papandreou C, Guillot A, Emmanouilides C, Slimane K, Kelkouli N, Kim S, Nguyen Tan Hon T. Efficacy and safety of sequential use of everolimus in patients with metastatic renal cell carcinoma previously treated with bevacizumab with or without interferon therapy: results from the European AVATOR study. Clin Genitourin Cancer. 2015; 13: 231-8.

28. Choueiri TK, Escudier B, Powles T, Tannir NM, Mainwaring PN, Rini BI, Hammers HJ, Donskov F, Roth BJ, Peltola K, Lee JL, Heng DY, Schmidinger M, et al. Cabozantinib versus everolimus in advanced renal cell carcinoma (METEOR): final results from a randomised, open-label, phase 3 trial. Lancet Oncol. 2016; 17: 917-27.

29. Motzer RJ, Escudier B, McDermott DF, George S, Hammers HJ, Srinivas S, Tykodi SS, Sosman JA, Procopio G, Plimack ER, Castellano D, Choueiri TK, Gurney H, et al. Nivolumab versus everolimus in advanced renal-cell carcinoma. N Engl J Med. 2015; 373: 1803-13.

30. Moch H, Artibani W, Delahunt B, Ficarra V, Knuechel R, Montorsi F, Patard JJ, Stief CG, Sulser T, Wild PJ. Reassessing the current UICC/AJCC TNM staging for renal cell carcinoma. Eur Urol. 2009; 56: 636-43.

31. Eisenhauer EA, Therasse P, Bogaerts J, Schwartz LH, Sargent D, Ford R, Dancey J, Arbuck S, Gwyther S, Mooney M, Rubinstein L, Shankar L, Dodd L, et al. New response evaluation criteria in solid tumours: revised RECIST guideline (version 1.1). Eur J Cancer. 2009; 45: 228-47.

32. Kim SH, Park WS, Kim SH, Joung JY, Seo HK, Lee KH, Chung J. Systemic treatments for metastatic renal cell carcinoma: 10-year experience of immunotherapy and targeted therapy. Cancer Res Treat. 2016; 48: 1092-101. 